\title{
TTR
}

Traduction, terminologie, re?daction

\section{Psychanalyse et traduction : voies de traverse}

\section{Ginette Michaud}

Volume 11, numéro 2, 2e semestre 1998

Psychanalyse et traduction : voies de traverse

Psychoanalysis and Translation: Passages Between and Beyond

URI : https://id.erudit.org/iderudit/037332ar

DOI : https://doi.org/10.7202/037332ar

Aller au sommaire du numéro

\section{Éditeur(s)}

Association canadienne de traductologie

\section{ISSN}

0835-8443 (imprimé)

1708-2188 (numérique)

Découvrir la revue

\section{Citer cet article}

Michaud, G. (1998). Psychanalyse et traduction : voies de traverse. TTR, 11(2), 9-37. https://doi.org/10.7202/037332ar

\section{Résumé de l'article}

Psychanalyse et traduction : voies de traverse - L'auteure présente les diverses voies d'approche abordées dans cette livraison, de la traduction du texte psychanalytique, aux transferts du traducteur freudien, en passant par la psychanalyse en traduction (lorsque la psychanalyse se fait elle-même opération traduisante entre les discours). À partir de réflexions en forme d'associations libres, elle interroge également certaines positions de Jean Laplanche, J.-B. Pontalis et Jacques Derrida qui éclairent les échanges se produisant entre psychanalyse et traduction.
Tous droits réservés (C TTR: traduction, terminologie, rédaction — Les auteurs, 1998
Cest protégé par la loi sur le droit d’auteur. L’utilisation des services d'Érudit (y compris la reproduction) est assujettie à sa politique d'utilisation que vous pouvez consulter en ligne.

https://apropos.erudit.org/fr/usagers/politique-dutilisation/ 


\title{
Psychanalyse et traduction : voies de traverse
}

\author{
Ginette Michaud
}

Chaque fois la traduction poétique et l'interprétation psychanalytique frayent, l'une pour l'autre, une voie nouvelle, s'orientent l'une l'autre sans aucun privilège unilatéral. Le travail de l'écriture poétique (traduisante) suppose la lecture psychanalytique, dans sa précision singulière et dans la généralité de ses lois [...]. Mais la traduction poétique n'applique pas, ne vérifie pas, ne suit pas, elle appartient au déchiffrement analytique dans sa phase la plus active et la plus inaugurale.

Jacques Derrida, "Fors „, dans Nicolas Abraham et Maria Torok, Cryptonymie. Le Verbier de l'Homme aux Loups, Paris, Flammarion, * Champs », 1976, p. 47.

[...] Ce livre essentiel, le seul livre vrai, un grand écrivain n'a pas, dans le sens courant, à l'jnventer, puisqu'il existe déjá en chacun de nous, mais à le traduire. Le devoir et la tâche d'un écrivain sont ceux d'un traducteur.

Proust, le Temps retrouvé, T. IV, p. 464.

La conjonction liant ces deux champs de la psychanalyse et de la traduction est-elle moins problématique, plus assurée aujourd'hui qu'elle l'était il y a une quinzaine d'années, au moment où le regretté François Peraldi - à qui nous voudrions rendre ici hommage - soulignait dans sa présentation d'un numéro de Meta consacré à ce même sujet à quel point le rapprochement - mais surtout une coordination, une articulation véritables - entre deux domaines en apparence aussi hétérogènes paraîtrait incongnu, pour des raisons différentes, tant à certains traducteurs qu'à bon nombre de psychanalystes ? Peraldi croisait alors le fer, non sans une évidente ironie polémique, avec ceux qui, dans chacun de ces 
groupes, envisageaient leur travail comme une « simple opération technique ${ }^{1}$ " consistant à faire passer, qui le signifiant, qui le symptôme, d'un système 1 à un système 2 , d'une langue de départ à une langue d'arrivée $e^{2}$ quitte justement à laisser se perdre en chemin cela seul qu'il importait de traduire et qui y résistait bien entendu de manière irréductible. Pourtant, au-delà d'une telle polarisation quelque peu caricaturale, d'ailleurs rapidement abandonnée par Peraldi, il y avait bien lieu d'insister au contraire sur le matériau commun aux deux pratiques et qui les liait de manière aussi profonde qu'intime : « le langage du sujet humain " et les remaniements subjectifs qui pouvaient s'operer, en psychanalyse comme en traduction, * par les seuls effets de la parole ${ }^{3}$. À relire cette livraison de Meta, on sera d'ailleurs frappé non seulement par la pertinence et l'actualité des aspects théoriques et pratiques examinés dans ce numéro, mais plus encore par l'agencement, le montage des textes retenus par Peraldi ${ }^{4}$. De fait, les titres des différentes rubriques — " La traduction du texte psychanalytique ", "La traduction dans le discours psychanalytique $»$, "L'interlangue en psychanalyse ", " Lecture psychanalytique et traduction du texte littéraire $n$ - frayent encore pour une large part notre manière de penser aujourd'hui ces rapports entre psychanalyse et traduction, et ils pourraient toujours servir de balises pour distinguer les différents points de vue adoptés par les collaboratrices et collaborateurs du présent numéro, où il s'agira tout aussi bien de suivre

' Francois Peraldi, Meta, « Psychanalyse et traduction *, 27:1, mars 1982, p. 9.

${ }^{2}$ A l'encontre de cette vue simpliste, Jean Laplanche, psychanalyste et lui-même traducteur professionnel de l'ceuvre freudienne, a souvent souligné la réversibilité, ta mobilité qui font l'essentiel du mouvement traductif : * Une traduction ne peut opérer qu'à partir de signes qu'elle retranscrit. Langue d'origine et langue cible, chaque système est à la fois les deux : cible pour celui qui précède, origine pour celui qui suit " (" Traumatisme, traduction, transfert et autres trans(es) ", dans $L e$ primat de l'autre en psychanalyse. Travaux 1967-1992, Paris, Flammarion, * Champs , 1997, p. 268).

${ }^{3}$ François Peraldi, art. cité, p. 11.

4 Une part importante du numéro était constituée de textes qui avaient paru antérieurement (c'était le cas pour ceux de Marthe Robert, Jean Laplanche et J.-B. Pontalis, Jean Allouch) ou avaient été * détournés * d'une parution imminente (l'extrait de * Moi - La psychanalyse * de Jacques Derrida). 
à la trace certains effets de l'inconscient à l'ceuvre dans la traduction du texte freudien que de la psychanalyse comme mode (modèle ?) traductif. Si par leur dispositif les textes de Meta suggéraient une progression, voire une intériorisation croissante dans le traitement du sujet (le changement des marqueurs de relation est à ce titre révélateur), l'énoncé de départ du présent numéro de $T T R$ a plutôt été saisi dans son double mouvement, de manière à mettre en valeur les points de contact et de passage entre la psychanalyse et la traduction. La composition du numéro reflète en effet ce double versant. D'une part, en tout bien tout honneur, les questions liées à la traduction $d u$ texte psychanalytique - et tout particulièrement du texte princeps, celui de Freud ${ }^{5}-$ y sont toujours bien présentes, réouvrant à partir de tensions, de raidissements, de * corrections $n$ quand ce n'est d'erreurs ou de contresens, une a clinique de la traduction freudienne ${ }^{6}$ " qui garde toujours son actualité. D'autre part, plusieurs collaborateurs ont également souhaité ne pas limiter leur approche à ces enjeux, aussi importants soient-ils, et considérer aussi l'autre versant de la question, soit la psychanalyse comme traduction car, on le sait, la psychanalyse dès ses commencements s'est elle-même élaborée comme un singulier modèle traductif. Ainsi entre psychanalyse et traduction, les voies de traverse sont-elles nombreuses et non moins importantes parce qu'elles sont plus obliques, du moins à première vue, ou indirectes (je pense ici aux textes de Christie McDonald et de Simon Harel tous deux particulièrement sensibles, dans leur lecture respective du passage à l'autobiographie chez Sarah Kofman ou des « transferts " imaginaires et secrets entre Beckett et Bion, à la psychanalyse comme opération traduisante entre fiction et théorie). De fait, si l'on cherchait à saisir ce qui s'est đéplacé dans ce champ de recherche, on pourrait suggérer que la préposition * entre n offre un indice intéressant de la circulation, du trafic qui se produit aux bords, aux marges ou frontières des deux domaines, de même que l'infléchissement, particulièrement significatif đans ce numéro, de la perception touchant la figure du traducteur. Souvent stigmatisé dans le passé (et non sans raison) pour ses fautes et lapsus, voici que le traducteur suscite dans plusieurs textes bien autre chose que les

\footnotetext{
${ }^{5}$ Mais également de Lacan bien entendu : Denise Merkle évoque dans ces pages quelques effets relevant de différences culturelles qui ont pu faire obstacle à la réception de Lacan dans les milieux anglo-saxons, montrant comme le danger de refoulement menace toujours dans l'acte de traduction.
}

${ }^{6}$ J'emprunte cette expression à Jean Laplanche (op. cit., p. 243). 
habituelles admonestations. Sans doute les réflexions de Walter Benjamin et d'Antoine Berman ou encore, dans le champ psychanalytique, celle de J.-B. Pontalis, sont-elles pour quelque chose dans la compréhension beaucoup plus complexe et nuancée que nous avons de la " tâche " du traducteur : j'y reviendrai plus loin. Par ailleurs, on peut aussi noter une autre différence sensible dans l'approche du sujet, du numéro de Meta à cette livraison de TTR: l'importance stratégique que prend, en tiers entre la psychanalyse et la traduction, le texte littéraire, dont la présence est loin d'être réductible au " cas ", à l' * exemple " ou, encore moins, à la seule " illustration". Dans le numéro de Meta, cet aspect, relégué dans la quatrième rubrique (de loin la moins développée), apparaissait presque comme une sortie, une transgression des limites du sujet, alors que, sans que cela ait été concerté, le texte littéraire s'est ici imposé d'emblée comme un important foyer de réflexion, comme si la scène d'écriture, que ce soit chez Alice James, Beckett, Celan, Kofman, Cixous et Derrida, offrait un indispensable point d'ancrage pour penser les rapports nouant l'une à l'autre la psychanalyse et la traduction. Je me garderai d'en tirer une proposition générale, mais il me semble qu'il n'y a nul hasard dans cette convergence, pas davantage d'ailleurs que dans la nature même de l'expérience traumatique convoquée avec tant d'insistance dans ces scènes d'écriture : l'intraduit n'aurait-il pas toujours à voir, d'une certaine manière, avec I'infigurable? Et n'est-ce pas cet intraduisible qui exige précisément de l'être et relance avec tant de force la pulsion de traduire chez le traducteur?

Sans vouloir dresser un bilan ou même un état présent des principaux travaux qui ont marqué le champ des études freudiennes sur cette question de la traduction, il serait difficile de ne pas au moins faire droit à quelques faits saillants. Ainsi en va-t-il, même si elle ne figure pas en tant que telle dans ce numéro, de l'entreprise collective qui a consisté à retraduire systématiquement toute l'ceuvre de Freud dans un a français

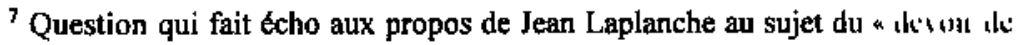
traduire $*$ : $\propto$ Le devoir de traduire, son Trieb inéluctable, ne provient pas ilu sus. la pulsion de traduire - là je commente à ma façon — vient d'une certaule là̧ u॥ de l'intraduisible. Encore une fois, c'est un devoir qui ne surgit pas du récéju'u c'est un impératif qui lui est apporté par l'œuvre elle-même; c'est un แII|'র'talıl catégorique : "Tu dois traduire parce que c'est intraduisible" $\times($ op. chl lin, 
freudien ", vaste projet mis en chantier en 1980 qui s'est poursuivi aux Presses Universitaires de France (dix volumes ont paru depuis 1989). À défaut de faire l'unanimité autour de ses principes, et encore moins autour de ce que cette équipe de traducteurs nomme de bien plate manière "Le style et son rendu ", ce projet, en bonne voie de réalisation une décennie plus tard, a tout au moins eu l'heur de nourrir des débats vifs autour de la traduction du texte freudien en France, oủ elle a toujours donné lieu à des conflits d'interprétations (ce qui la distingue déjà d'autres milieux " nationaux ": la traduction du texte freudien en anglais n'a pas, à ma connaissance, déclenché de telles passions; il serait intéressant de se demander un jour sérieusement pourquoi, au-delà d'une ethnopsychanalyse sauvage ${ }^{8}$ ). Sous la direction d'André Bourguignon, Pierre Cotet, Jean Laplanche et François Robert, cette équipe de traducteurs a donc, on le sait, mis en ceuvre une certaine conception de la traduction qui, depuis la suppression de certains termes qui s'étaient imposés en français depuis cinquante ans et leur remplacement ${ }^{9}$, la fabrication de nombreux néologismes ${ }^{10}$ et un maniement uniformisant du

'Jean Laplanche a, à l'occasion, esquissé quelques hypothèses, extrêmement rapides, sur la question, notant, à la suite d'Antoine Berman, que l'approche française à la traduction en général est sans doute plus ethnocentrique, autocentrique que l'approche allemande, qui procède selon * un mouvement qui veut que la traduction, loin de rester en soi et de rapprocher l'autre de soi, soit un aller vers l'autre ^ (op. cit., p. 292).

9 Par exemple, souhait pour désir, fantaisie pour fantasme, négation pour dénégation, souvenir-couverture pour souvenir-écran, mise à mort du pere pour parricide, et bien d'autres. Voir l'article * Traduction * (auquel j'emprunte ces exemples), dans Élisabeth Roudinesco et Michel Plon, Dictionnaire de la psychanalyse, Paris, Fayard, 1997, p. 1066.

10 Citons ceux qui ont levé le plus de... résistances : désirance, animique, frustrané, désaide, retirement, vicarier, refusement, rétrofantasier, etc. Laplanche s'est expliqué à plusieurs reprises sur sa conception du modèle traductif qui repose, entre autres, sur ces principes : le devoir inéluctable de traduire, le refus du * refoulement * (par rapport à l'intraduisible), la stricte délimitation des rapports entre commentaire et traduction, la constance impérative d'un terme pour tous les concepts majeurs indépendamment de tel contexte particulier, le rôle dévolu au néologisme ( modèle privilégié pour concevoir le processus de sublimation : le néologisme est lancé pour être habité des deux côtés à la fois : habité par l'usage de la langue cible, mais aussi habité par la pulsion venant de la langue d'origine et peut-être de plus loin qu'elle », op. cit., p. 305). 
lexique ne tenant pas compte du contexte et de l'évolution de la pensée de Freud, s'est attirée de nombreuses critiques. Je n'ai pas l'intention de reprendre ici les arguments invoqués par les uns et les autres dans ce débat ${ }^{\mathrm{II}}$, mais je crois qu'il est important de rappeler cet événement éditorial (ou, tout au moins, médiatique) pour souligner à quel point " Cent Ans après » la naissance de la psychanalyse l'œuvre de Freud attend toujours en français une édition complète et sûre, alors que le monde anglo-saxon a pu s'appuyer depuis plusieurs décennies déjà sur l'édition systématique de la Standard Edition (toute banalisante soit-elle à d'autres égards) ${ }^{12}$. A l'évidence, Freud ne " passe " pas de la même façon d'une langue à l'autre (peut-être même ne dit-il pas tout à fait la même chose en allemand et dans ses langues de traduction), et s'engage là un tout autre débat que celui portant sur les seules défaillances de ses traducteurs. Patrick Mahony nous rappelle d'ailleurs cette situation à propos de la parution en 1996 de la monumentale Konkordanz allemande des Gesammelte Werke, autre coup d'éclat qui a récemment marqué le champ des études psychanalytiques. Or il n'est que de comparer, comme

" J'ai eu l'occasion de développer ailleurs cette discussion critique : voir $\propto$ Freud : N.D.T. *, Études françaises, 24 :3, 1988, p. 125-140 et * Freud : N.D.T. ou Affects et fantasmes chez les traducteurs de Freud *, $T T R$, II : 2, 1989, pp. 105-127.

12 Rappelons que l'on compte à ce jour, outre la toute-puissante traduction anglaise de Strachey, quatre éditions intégrales de l'œuvre freudienne : en espagnol (Obras completas, 22 vol., Buenos Aires, Amorrortu, 1922-1978, traduction initiée par José Ortega y Gasset et reprise par Luis Lopez Ballesteros et Rosenthal), en italien (Opere di Sigmund Freud, 12 vol., Turin, Boringhieri, 1967-1980, projet mené par Cesare Musatti et plusieurs traducteurs), en portugais (Edição Standard Brasileira das obras completas de Sigmund Freud, São Paulo, Editora-Imago, 1970-1977) et en... japonais. Par ailleurs, la situation du corpus freudien est loin d'être simple dans sa propre langue \& maternelle *, l'allemand : sur la double édition des ceuvres de Freud (les Gesammelte Schriften de Vienne et les Gesammelte Werke de Londres) et la disparition de son ceuvre des maisons d'édition sous le régime nazi, voir Ilse Gubrich-Simitis, " Histoire de l'édition des ceuvres de Freud en langue allemande , Revue internationale d'histoire de la psychanalyse, 4, 1991, pp. 13-71. Devant la pauvreté du travail éditorial en allemand, Ilse Gubrich-Simitis entreprit au début des années soixante une nouvelle édition des Guvres complètes de Freud, mais il n'existe toujours en langue allemande qu' « une édition critique de textes choisis, les Studienausgabe, et une édition intégrale mais non critique, les Gesammelie Werke " (Elisabeth Roudinesco et Michel Plon, * Traduction *, op. cit., p. 1064). 
le fait de manière suggestive Mahony, la Konkordanz allemande et la Concordance anglaise qui accompagnait jusqu'ici l'édition de Strachey pour s'apercevoir de discordances révélatrices sur le plan lexicologique ${ }^{13}$. Les voies associatives ne s'ouvrent visiblement pas du tout de la même façon et ne suivent pas les mêmes chemins d'une langue à l'autre, et Mahony a beau jeu de le montrer a partir de quelques exemples idiomatiques singulièrement éloquents prouvant, si besoin était, cette vérité éprouvée par tous les traducteurs du texte freudien : une question d'ordre lexicologique n'est jamais chez Freud un simple problème de terminologie. L'usage de la Konkordanz met en effet rapidement en lumière l'incidence des processus de l'inconscient et du préconscient jusque dans la syntaxe de Freud — car cet ouvrage a l'avantage sur son prédécesseur d'offrir également au lecteur le contexte des différentes occurrences lexicales -, et il permet, entre autres, de suivre à la trace d'autres trajets de mots, d'autres aiguillages et associations verbales dans l'œuvre de Freud, de laisser émerger certains termes occultés dans la Concordance, de remettre en circulation ce qui avait été figé et censuré dans la Standard Edition. La Konkordanz s'avère ainsi non seulement un précieux outil de référence mais encore un véritable instrument de pensée incitant les chercheurs à entreprendre, comme le suggère Mahony, * une étude des diverses tendances en psychanalyse à travers les frontières nationales et linguistiques ${ }^{14}$ " et même éventuellement à remettre en

${ }^{13}$ Déjà en 1967, Laplanche (c'était bien avant le programme de la retraduction) et J.-B. Pontalis constataient qu'il fallait, dans le cas du texte freudien, \& se colleter des années avec un auteur pour repérer les pièges innombrables qu'offre non seulement $l a$, mais $s a$ traduction. On ajoutera que ces pièges ne sont pas seulement d'ordre lexicologique : le meilleur germaniste, même muni d'un code exhaustif et raisonné des principaux concepts, risque de multiplier encore les erreurs et les contresens s'il n'est pas au sens plein et avant tout un "freudologue" " (Le Monde, $1^{\text {er }}$ mars 1967).

${ }^{14}$ Voir en ces pages Patrick J. Mahony, * Discordance dans la concordance : ressacs de la traduction $n$, p. 49. 
question l'hégémonie ${ }^{15}$ exercée par la Standard Edition qui a imposé I'anglais comme la lingua franca de la psychanalyse depuis la Seconde Guerre. La comparaison même rapide de la Konkordanz et de la Concordance confirme en tout cas de manière toute concrète que, même à ce niveau relativement élémentaire (non pas au sens de simple, mais de fondamental, de ce qui, en grammaire ou en mathématique, permet des combinaisons infinies), les discussions déclenchées par la forme même des mots et concepts freudiens ne relèvent jamais seulement d'une " simple technicité * : comme l'affirme également Jean Laplanche dans un autre contexte, « [...] avec les problèmes de traduction nous sommes au cœur des problèmes de la psychanalyse ${ }^{16}$ ". C'est que la psychanalyse opère sans cesse une traduction originaire en quelque sorte, une conversion anasémique, dans son rapport au mot et plus généralement à la langue et à la tradition des concepts, comme l'ont bien vu Nicolas Abraham et Jacques Derrida : transitant en apparence par des mots ou figures domestiques, elle introduit par effraction ou détournement un " changement sémantique radical ${ }^{17}$ " dans le langage, elle * compose avec toutes sortes de résidus ${ }^{18}$ "qui viennent des anciens noms ou des concepts hérités, bref, * cette étrangeté habite les mêmes mots, se déguise dans la

${ }^{15}$ Louée pour sa cohérence et sa rigueur, la traduction anglaise de Strachey a souvent été mise en cause parce qu'elle rendait l'appareil conceptuel freudien plus technique (en latinisant par exemple nombre de concepts) et surtout parce qu'elle aplatissait le style littéraire de Freud, ce qui entraîna des conséquences plus sérieuses dans le transfert que développèrent plusieurs générations de lecteurs et de psychanalystes à la parole du * Maître ${ }^{\prime}$. Pour une critique bien documentée de l'édition anglaise, voir Riccardo Steiner, * Une marque internationale universelle d'authenticité. Quelques observations sur l'histoire de la traduction anglaise de l'ceuvre de Sigmund Freud, en particulier sur les termes techniques *, Revue internationale d'histoire de la psychanalyse, 4, 1991, pp. 71-188.

I6 Jean Laplanche, "Spécificité des problèmes terminologiques dans la traduction de Freud ", op. cit., p. 307.

${ }^{17}$ Jacques Derrida, "Fors ", dans Nicolas Abraham et Maria Torok, Cryptonymie. Le Verbier de l'Homme aux Loups, Paris, Flammarion, „Champs \#, 1976, p. 44.

is Ibid., p. 44. 
même langue et le même système discursif ${ }^{9}$ n. Cette conversion anasémique opérée par la psychanalyse dans son rapport au langage est également présente dans l'équivoque sans cesse renaissante des mots et concepts de cette Konkordanz freudienne, dérobés à un espace discursif traditionnel pour traduire l'impensé, ce qui lui échappe par essence.

Au delà de ces aspects lexicographiques (qui, déjà, peuvent nous mener loin), à quoi tient cette intimité de la traduction et de la psychanalyse ? Car il est indéniable que dès les commencements de la psychanalyse, dès les tout premiers textes de Freud ${ }^{20}$ en fait, s'est établi un " rapport intime de la traduction elle-même avec l'objectif de la psychanalyse, qui est tentative de traduction de l'inconscien ${ }^{21}$ ". Dans une lettre à Fliess datée du 6 décembre 1896, Freud compare déjà l'appareil psychique à une série d'inscriptions successives dont chacune traduit la précédente dans un idiome différent ${ }^{22}$ " et il assimile de manière saisissante le refoulement à un refus de traduire, à ce qui bloque le mouvement de détraduction/retraduction qui doit pouvoir se poursuivre dans le travail de l'analyse. Mais la traduction, déjà à ce moment, n'est pas seulement ce qui permet de penser, en pratique, ce qui se passe dans la cure, elle informe et travaille au plus près, au corps serait-on tenté de dire, les concepts majeurs de la psychanalyse, notamment ceux

${ }^{19}$ Ibid., p. 44.

${ }^{20}$ On rappellera que l'intérêt de Freud envers la traduction ne fut pas que théorique, mais qu'il s'y intéressa aussi dans sa jeunesse comme praticien, puisqu'il fut lui-même le traducteur de textes de Stuart Mill et de Charcot. Il est amusant de voir Freud comme l'un de ces traducteurs * à la française n tant honnis par Laplanche à cause des libertés qu'ils se permettaient. Freud préconisait en effet * de lire tout une page ou tout un passage de l'original, puis de fermer le livre et d'écrire, en se demandant : comment rendre cela en allemand ? (cité par Laplanche, op. cit., 292). Avec une approche aussi peu orthodoxe et non systématique, Freud n'aurait certes pu faire partie de l'équipe de traducteurs qui s'est penchée sur son propre texte !

${ }^{2 !}$ Jean Laplanche, op. cit., p. 315.

${ }^{22}$ Ibid., p. 307. 
d'interprétation et de transfert (Übertragung qui, soulignons-le, peut se traduire en français par * traduction ") qui lui est étroitement lié. Or il est intéressant de remarquer avec Catherine Mavrikakis que Freud doit beaucoup, sur ce terrain aussi (sans pleinement le reconnaître), à la figure de l'hystérique. Car ce n'est pas le hasard qui le conduit à élire comme " modèle de la constitution de l'inconscient la traduction et ses ratés ${ }^{23}$ ", mais bien l'expérience de la cure avec les patientes hystériques : la figure d'Anna $O$. se révélera à cet égard particulièrement importante, puisque toute son analyse se joua littéralement à travers le passage de sa langue maternelle (l'allemand dont elle perdit l'usage) à une langue étrangère (l'anglais) qui, en venant à sa rescousse, permit du coup de traduire le transfert. Perte de la langue maternelle, recours à une langue Autre, déplacement qui opère la conversion du symptôme : cette scène "primitive " de la traduction au cours de laquelle Anna $O$. jouit d'un extraordinaire don des langues, Freud n'allait pas l'oublier. Même s'il interprétera sur le plan clinique cette remarquable capacité de trađuction de l'hystérique de façon quelque peu réductrice, n'y voyant qu'un symptôme, c'est-à-dire une " mauvaise " traduction qui doit être ramenée après les détours et boucles de l'analyse à la langue d'origine, Freud se souviendra de cette expérience et en tirera une certaine manière de penser la psychanalyse avec la traduction. Comme le note Catherine Mavrikakis, Freud accordera toujours en effet par la suite une place fondamentale aux images de la traduction dans sa conceptualisation des processus inconscients, notamment dans l'étiologie de l'hystérie qu'il définira précisément comme un a défaut de traduction ". En ce sens, il n'est pas exagéré de dire que la psychanalyse, dès l'origine, va se penser comme "passage, [...] déplacement d'un affect à l'autre, de représentations non verbales à verbales, d'affects à signes. Ce déplacement est chez Freud là où tout commence. En fait, il n'y aurait pas de théorie psychanalytique sans cette notion essentielle qui est la possibilité fondamentale de pouvoir transformer et traduire une chose dans une autre $\mathrm{e}^{24}$.

C'est donc dire à quel point la psychanalyse est inséparable d'une pensée de la traduction. Et cela même si Freud ne la désigne pas en

${ }^{23}$ Ibid. p. 253. C'est l'auteur qui souligne.

${ }^{24}$ Voir, dans ce numéro, Catherine Mavrikakis, * L'hystérique face aux symptômes de la traduction ", p. 76. 
tant que telle comme la fondation de la psychanalyse. Il faut en effet souligner ce paradoxe : on ne trouve pas dans l'cuuve freudienne une réflexion théorique systématique sur la traduction, celle-ci demeure finalement un processus non complètement approprié, qui ne trouve pas sa place dans la théorie psychanalytique. Cette situation est d'autant plus curieuse et significative que l'intérêt de Freud pour cette question ne se limite pas, tant s'en faut, à ses premiers textes, mais traverse de part en part toute son œuvre jusqu'aux tout derniers ouvrages. De fait, $L$ 'Homme Molse est-il autre chose qu'une interrogation sur la traduction, alors que Freud cherche à établir, comme le rappelait récemment Fethi Benslama, que $*$ le nom de Moïse est une hébraïsation de l'égyptien ", autrement dit que " le nom propre du fondateur est non propre aux hébreux et qu'il est le produit d'une traduction "2s " S'il est indéniable que la psychanalyse a eu recours à la traduction pour se fonder, s'il est tout aussi évident que l'originalité - son trait le plus originaire ? - de la pensée freudienne tient précisément dans une démarche qui procède par une série de translations, il paraîtra d'autant plus surprenant que le statut psychique de la traduction n'ait pas été complètement pris en compte et que la tâche de penser la traduction comme fondatrice de la psychanalyse, comme une opération qui ne cesse de se produire en son sein comme " l'étrangement originaire ", soit toujours la nôtre. Est-ce précisément, comme le remarque encore avec justesse Benslama, parce que cette question est l'évidence même qu'elle n'a pas vraiment été traitée en propre autant qu'elle aurait da l'être ${ }^{26}$ ? On pourrait le croire, on pourrait même suggérer que si cette nécessité d'une réflexion théorique sur la traduction, " ni Freud, ni la

${ }^{25}$ Fethi Benslama, "La répudiation originaire ", Cahiers Intersignes, * Idiomes, nationalités, déconstructions. Rencontre de Rabat avec Jacques Derrida \#, $\mathbf{n}^{\circ} \mathbf{1 3}$, automne 1998, p. 120. Dans L'Homme Moüse qui, notons-le, retraduit le mythe scientifique de Totem et tabou, Freud est pourtant au cour de la question, operant une série de traductions, qui vont du mythe à la métapsychologie, • de l'enfant dans le nom de Moïse, à la figure psychique de l'infantile, et du mythe de la naissance du héros, au roman familial; aboutissant à cette configuration historique de l'origine approchée à travers une écriture qualifiée de romanesque. [...] Cette relation entre l'infantile, l'étranger et la fiction relève d'une articulation princeps concernant le statut psychique de la traduction * (ibid.).

${ }^{26} \mathrm{Ibid}$. Comme l'observe Benslama, " [...] dès lors que l'étranger est à l'origine, le principe de son épreuve dans le langage est inévitablement la traduction ". 
pensée psychanalytique à sa suite, ne l'ont éprouvée, ni relevée ${ }^{27}$, cela n'est pas sans rapport avec les transferts complexes des traducteurs ultérieurement aux prises avec cet impensé à l'œuvre dans leur propre traduction du texte de Freud.

*

Les transferts des traducteurs ont depuis longtemps retenu l'attention des critiques, ils ont même, il n'est pas exagéré de le dire, exercé une certaine fascination, en laissant parfois affleurer au détour d'une humeur " mal métabolisée ${ }^{28}$ ", d'un " état d'âme " (ces " états d'âme * justement tant décriés par Laplanche) insuffisamment neutralisé, les fantasmes engagés à leur insu ou non dans l'acte de traduction. Catherine Mavrikakis montre bien comment certaine * éjaculation de la pensée $n$ confiée par Alice James à son joumal était impossible à entendre de la part de sa traductrice qui, soumise à l'idéologie féministe, ne put s'empêcher de détourner cette affirmation * phallique " de l'hystérique en signe de faiblesse, retoumant dans sa traduction Alice James à la position de victime souffrante dont elle tentait précisément de s'affranchir par l'écriture. Et les exemples de tels contresens inconscients majeurs ne sont pas, hélas, rares, ils seraient bien plutôt inéluctables, car de l'altérité transformante, plus ou moins assumée, à l'altération déformante, la ligne est souvent bien mince et facilement franchie dans le contre-transfert du traducteur...

De fait, si la traduction fait indissolublement partie du corps psychanalytique, cela implique tout aussi inévitablement sa contrepartie, à savoir qu'il est impensable de traduire sans l'inconscient, sans que ses effets ne refluent dans la traduction. C'est sans doute l'une des raisons qui font des commentaires des traducteurs - qui se glissent notamment dans

${ }^{27}$ Ibid. L'exemple de Wilfred R. Bion, tel que l'analyse ici Simon Harel à travers notamment le a rêve de Babel o retenu comme expérience fondatrice de sa * fiction " théorique, constitue à cet égard un contrepoint des plus intéressants.

${ }^{2 R}$ Rappelons cette proposition de Laplanche qui vaut aussi pour le traducteur : - C'est en ce sens que le transfert peut et doit être confronté à cette traduction originaire par laquelie l'individu métabolise, non sans reste, les signifiants de son histoire individuelle " (op. cit., p. 253. C'est l'auteur qui souligne). 
ces fameuses notes infrapaginales laissant revenir le refoulé de la traduction - des lieux particulièrement intéressants pour saisir ces rapports transférentiels à l'œuvre dans l'opération traduisante. Car comment pourrait-il être indifférent ou sans conséquences qu'un traducteur utilise, pour décrire sa relation au texte de Freud, une métaphore relevant par exemple de la stratégie militaire, comme cela se produit chez Laplanche :

Traduire " selon le contexte ", traduire au courant de la plume, s'esbaudir devant le premier terme freudien aux allures \& abyssales ", parsemer la traduction de mots allemands, c'est capituler devant la tâche (et devant le plaisir) de pousser au plus loin la lutte avec le texte, le combat avec l'ange. Sans doute la défaite du traducteur est inéluctable : l'essentiel est de repousser toujours plus avant la frontière sur laquelle se signera l'armistice ${ }^{29}$ ?

Si cette métaphore belliqueuse peut déranger, l'image de l'opération chirurgicale qui décrit le geste traductif idéal (le Moi idéal du traducteur?) pour J.-B. Pontalis est-elle moins significative? Rappelons ce commentaire révélateur, qui a aussi frappé Monique Schneider ${ }^{30}$ par sa tonalité mélancolico-dépressive :

Un traducteur opère. Autant qu'il le sache et le veuille : la restitutio ad integrum n'est pas à sa portée. On dit que la qualité maîtresse du chirurgien est, à chaque instant, la décision. Décider, le traducteur ne fait que cela : le choix des mots, l'ordre des mots, l'agencement de la phrase, le rythme, l'accent porté sur telle conjonction, tel adjectif... Ne pas confondre une traduction " soignee $"$, médicalement prudente (primum non nocere...) et une traduction operée, la seule à pouvoir être opérante ${ }^{3 !}$.

${ }^{29}$ Ibid, p. 252.

${ }^{30}$ Voir dans ces pages Monique Schneider, “ Éprouver le passage », p. 58.

${ }^{31}$ J.-B. Pontalis, * Encore un métier impossible ", dans Perdre de vue, Paris, Gallimard, « Connaissance de l'Inconscient ", 1988, p. 198. Incidemment, le recours répété au latin, langue morte à laquelle est confié le vif du sujet, n'est pas sans faire penser aux dénégations de Freud concernant la chose sexuelle dans le cas Dora : l'intrusion de la langue étrangère, au moment de définir la tâche du traducteur, fait figure d'interdit. \& Primum non nocere... * : on ne saurait mieux dire et (ne pas) faire à la fois... 
Envisager de la sorte la traduction en termes d'agression sur le corps textuel de l'autre n'est certes pas innocent et déterminera toute une série de choix, volontaires et inconscients, comme ceux par exemple qui pousseront d'autres traducteurs de Freud, trop bien intentionnés, à pudiquement recouvrir dans leur traduction du célèbre rêve de "L'Injection faite à Irma " la * mâchoire * du père de la psychanalyse, pourtant bien saillante dans le texte de Freud, par de bienséants « maux de tête ". Monique Schneider analyse de manière très convaincante ce qui est en jeu dans de tels déplacements, qui visent à revoiler ce qui est alors exposé, de manière insupportable aux yeux des fils traducteurs, dans le texte freudien. Peut-être le mot * mâchoire " doit-il être effacé justement parce que les traducteurs se sont trop identifiés à ce chirurgien prudent fantasmé par Pontalis, et qui doit amputer le texte du corps étranger qu'il abritait. Monique Schneider suggère avec perspicacité que le mot " mâchoire " activait sans doute trop les associations en direction du corps du maître, donné à voir dans cette bouche souffrante dans une contiguïté jugée menaçante avec la gorge béante d'Irma. Cette perte de maîtrise sera donc déplacée dans un signifiant plus * neutre ", mais qui répète à son insu sur le corps du texte le bâillonnement dont il était question dans le rêve de Freud. On tient là un excellent exemple d'une traduction ne supportant pas l'étrangeté du texte freudien et qui coupe dès lors les associations inconscientes du lecteur, alors qu'une " bonne " traduction, une traduction moins refoulante, l'aurait gardé mobile et ouvert.

Mais revenons un instant encore aux métaphores relevées chez Laplanche et Pontalis à propos de la tâche du traducteur. Sans exagérer leur portée, il est possible de croire qu'elles révèlent une certaine façon de se situer, pour chacun de ces traducteurs (et ce ne sont pas les moins éclairés), par rapport au texte freudien. Chez Laplanche, on est carrément dans le registre de l'affrontement, du combat, de la guerre même; le mot " armistice " résonne en effet avec force, ne pouvant manquer de réactiver, à l'horizon de cette opération militaire où le traducteur est envoyé * au front ", le contentieux des guerres franco-allemandes, inévitablement réinscrites dans ces fantasmes sur les deux langues. Il s'agit pour le traducteur de ne pas céder un pouce de terrain, d'avancer coûte que coûte en territoire ennemi, de conquérir de haute lutte (et la guerre de religion, sacrée, n'est pas loin puisque surgit « spontanément " l'image biblique de Jacob luttant avec l'ange), par invasion ou par annexion donc, le texte

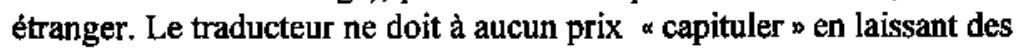
mots allemands intraduits, même si la * défaite » est inévitable. Il y aurait 
beaucoup à dire sur cette image agressive/défensive, qui pose le rapport au texte de Freud de manière bien peu psychanalytique (que penserait-on en effet d'un psychanatyste qui envisagerait en des termes aussi conflictuels l'expérience analytique et qui chercherait à maîtriser le transfert par la force?). Le désir de conquête, l'agression au coeur de cette image militaire lèvent certes le voile sur la violence inhérente à l'opération de traduction, mais il ne faudrait pas pour autant oublier, en toute bonne logique inconsciente, que cette image-écran peut également en dissimuler une autre, tout aussi importante, même si elle se fait plus secrète : au-delà du fantasme de domination, c'est en effet l'endeuillement qui affecte le traducteur vaincu, aux prises avec une impossible réparation symbolique, qui émerge de tous ces " il faut ", de tous ces impératifs catégoriques et directives prescriptives (Monique Schneider et Alexis Nouss consacrent ici des réflexions très riches à cet affect mélancolique).

Chez Pontalis, l'image est plus nuancée, complexe : le traducteur apparaît en chirurgien qui opère, coupe et greffe pour sauver ce qu'il peut du corps-texte, implicitement malade. L'agression inhérente à ce fantasme de traduction est toujours présente (le sadisme médical faisant encore une fois écho à la scène originaire du " Rêve d'Irma "), mais elle reste associée de manière subtile à travers la chaîne de signifiants $\alpha$ opérée/opérante n à l'idée d'œuvre. Contrairement à Laplanche qui considère la traduction exclusivement au service du texte (elle doit tout faire pour * s'effacer * et s'interdire toute " dérive " interprétative ou " divagations de l'herméneutique ${ }^{32}$ ), Pontalis ne dénie pas aussi catégoriquement au traducteur de pouvoir, au contact des mots du texte traduit, faire ceuvre à son tour. Si le traducteur a bien exécuté son travail, s'il a bien su découper, prélever, suturer le texte sur lequel il opère $"$, la traduction sera en retour opérante, c'est-à-dire qu'elle permettra à l'cuvre de se rejouer en elle, comme le suggère Daniel Sibony en évoquant aussi dans son texte le geste de traduire en termes de coupure-lien entre deux langues. Mais cette traduction-opération ne va pas sans mélancolie chez Pontalis, pour qui le traducteur, cet être entre-deux-langues, éprouve justement la perte des deux langues :

${ }^{32}$ Jean Laplanche, op. cit., pp. 252-253. 
Le traducteur, je le vois d'abord comme un être en souffrance : il a perdu sa langue sans en gagner une autre. [...] Le traducteur doit être doué d'une capacité infinie d'être triste : il n'a pas le droit de jouer de ses mots à lui, il n'a pas le pouvoir de restituer les mots de l'autre. Injustice de son sort : plus son intimité est profonde avec la langue étrangère, plus il demeure en elle, et moins il se sent les moyens de refranchir la frontière ${ }^{33}$.

On remarquera au passage que chez Laplanche la frontière n'existait que pour être " repouss[ée] toujours plus avant ", alors que pour Pontalis elle barre tout retour du traducteur à sa * langue d'origine * : une fois que le traducteur a passé la ligne, de sa langue dite * maternelle $*$ a une langue dite * étrangère ", il ne peut plus revenir à la première, il demeure suspendu dans l'entre-deux des limbes, pour évoquer le titre de son demier ouvrage, $L$ 'Enfant des limbes. Ce qui, en termes de fantasme autour de la frontière, métaphore obligée de la traduction, laisse entrevoir que le traducteur ne dispose pas du même jeu, qu'il ne navigue pas tout à fait dans les mêmes eaux...

* Le traducteur, dit Pontalis, doit être doué d'une capacité infinie d'être triste " : étrange prescription relevée par Monique Schneider, qui se demande avec rajson si ne jouerait pas dans l'imposition de cet affect mélancolique un déni de la jouissance secrètement éprouvée par le traducteur. Car sa * tâche * ne relève peut-être pas seulement du devoir surmoïque, comme le suggère l'image de Pontalis qui le voue à la perte et à l'insatisfaction, oscillant entre les rives des deux langues traversées sans possibilité de regagner l'une ou l'autre. Le traducteur dans les textes de Pontalis est saisi dans l'après coup du processus, « après Babel " (post coïtum triste), alors que les choses se présentent sous un tout autre jour si l'on remonte un peu en aval et qu'on s'attache plutôt à ce qui se passe au moment même du passage, c'est-à-dire au vif de l'échange, du mouvement de va-et-vient entre les langues. Monique Schneider et Daniel Sibony insistent tous deux, dans des perspectives différentes, sur cette fantasmatique, soulignant à quel point la traduction peut aussi être une expérience porteuse de jouissance. Mais pourquoi, demande Schneider, mettre tant d'insistance à déplorer, au sens fort de l'expression, la perte de langue, l' a être en souffrance ", si ce n'est pour voiler cette jouissance en jeu dans la traduction? En tout cas, il est clair que les lecteurs

${ }^{33}$ J.-B. Pontalis, op. cit., pp. 197-198. 
" reviseurs " - ceux qui se penchent sur les traductions pour y saisir les pulsions et résistances intriquées dans le processus - retirent de cet exercice de lecture au second degré un plaisir assez vif...

Que serait une a bonne " traduction du point de vue psychanalytique? Daniel Sibony nous donne un élément de réponse en suggérant que la traduction réussie serait celle qui serait capable de retraduire les « fautes signifiantes $n$ du texte original et qui parviendrait à retransmettre l'expérience de manque éprouvée par l'auteur. Cette définition serait assez proche des qualités reconnues par Laplanche à la traduction de la Bible faite par Chouraqui (également cité par Sibony) : " On pourrait dire que, d'une certaine façon, il traduit le grec pour y réinjecter (je ne peux pas dire autrement) le refoulé, c'est-à-dire le nontraduit dans le grec, cela aux fins d'essayer de traduire plus loin, de retraduire en réintégrant quelque chose qui a été laissé tomber, qui a été refoule ${ }^{34}$ ". La $~$ bonne " traduction serait donc celle qui parviendrait à déjouer, ne serait-ce qu'un instant, la censure et à retraduire le refoulé. Les traductions de Paul Celan - Alexis Nouss les analyse ici avec un tact admirable - sont au plus près de cette exigence et elles atteignent souvent elles-mêmes au poème. Abordant pour une unique fois dans son œuvre l'événement de la Shoah (partout présent ailleurs mais sans être nommé) à travers sa traduction du poème de Jean Cayrol, Nuit et brouillard, Celan s'avance ici aussi loin que possible en répondant à l'appel à traduire de l'intraduisible même, tout en faisant silence sur ce qui, de ce réel impossible, doit rester infigurable. L'affect mélancolique qui imprègne cette écriture de la traduction est d'une tout autre profondeur, est-il besoin de le dire?, que celui qui touchait le traducteur de Pontalis. Celui-ci ne parvenait pas à faire son deuil, oscillant entre le désenchantement et le triomphe maniaque, alors que Celan traducteur doit se mesurer à une tout autre épreuve : il doit faire le deuil du deuil, c'est-àdire élaborer le travail psychique de la perte au cour même de la traduction. Le transfert traductif accomplit dès lors une double tâche : traduire la perte en la figurant -- ce qui est impossible quand l'objet, comme c'est le cas ici, manque à jamais de manière aussi radicale - et la compenser tout à la fois. Comme le voit bien Nouss dans cette

\footnotetext{
${ }^{34}$ Jean Laplanche, op. cit., p. 299. Je souligne.
} 
expérience limite où la traduction se fait elle-même réécriture du trauma, retouche de " ce qui n'est pas en mots, [de] ce qui est sous les mots ${ }^{35}$, * la traduction va produire des énoncés absents ailleurs [...] dût-elle inventer dans l'original, et d'autre part, elle va omettre ou transformer certains énoncés, témoignant d'une résistance révélant des interdits ${ }^{36}$ \%. Dans le même temps, la traduction avoue l'impossibilité du dire, elle refuse le deuil, et elle trouve quand même $\alpha$ le courage de nommer ce que l'original tait ${ }^{37}$, elle donne voix et affect à la blessure non symbolisable.

Les choix de Celan révèlent un traducteur travaillant au plus près des processus primaires - condensation, déplacement -, et ils transmettent, en encryptant dans le texte son propre témoignage silencieux, le devoir double, « à plus d'un ", de respect et de trahison dont parle si bien Derrida lorsqu'il donne sa propre interprétation de la tâche du traducteur :

${ }^{35}$ Pontalis, op. cit., p. 198. L'expérience celanienne de la traduction remet complètement en question cette affirmation de Pontalis qui, à nouveau, interdit au traducteur de pouvoir toucher aux mots et lui assigne l'impuissance : "Venant après, operant sur un texte déjà produit, elle [la traduction] ignore ce temps où les mots se cherchent et parfois se trouvent pour dire, pour toucher, ce qui n'est pas en mots, ce qui est sous les mots. Le traducteur, par position, ne prend pas part a ce mouvement qui fait l'écriture : il est réduit à mettre des mots à la place d'autres mots $[\ldots]$. Le traducteur $[\ldots]$ est soumis sans pouvoir échapper à sa condition, celle d'un scribe des énonces qui a renoncé à atteindre, même par procuration, la place, il est vrai toujours incertaine, d'un sujet d'énonciation * (op. cit., p. 198199). Celan traducteur transgresse constamment cet interdit du toucher, ce Noli me tangere. et assume dans sa traduction le "mouvement qui fait l'écriture " (de Cayrol et de la sienne propre) et une place, fut-elle absentée, d'un * sujet d'énonciation ". De manière éclatante, il n'est pas que le * scribe des énoncés ", pas plus que, comme le laisse croire la temporalité encore phénoménologique utilisée par Pontalis, il ne vient simplement après : la traduction de Celan n'opère pas sur "le texte déjà produit " (ce qui, en soi, implique déjà une certaine conception réductrice de ce qu'est un texte), elle cherche ses mots comme Cayrol l'a fait, elle est dans ce temps de l'inconscient, si bien nommé ailleurs par Pontalis : ce temps qui ne passe pas.

${ }^{36}$ Voir dans ce numéro Alexis Nouss, " La traduction mélancolique (sur Paul Celan) », p. 207.

${ }^{37}$ Ibid., p. 215. 
Ma tentation - aujourd'hui comme toujours, dans doute - me dicterait deux fidélités : de respecter l'irréductibilité intraduisible de l'idiome, certes, mais en même temps d'appréhender autrement cette intraductibilité. Celle-ci ne serait plus une limite hermétique, l'opacité impénétrable d'un écran, mais bien au contraire une provocation à la traduction. Déjà un engagement à tradurire dans l'expérience de l'intraduisible comme tel. Appréhender l'intraduisible, l'appréhender comme tel, c'est lire, c'est écrire, au sens fort du mot, bien sûr, c'est le corps-à-corps avec l'idiome; mais c'est donc dejjă une épreuve, la première épreuve de l'appel à tradurire ${ }^{38}$.

Ce devoir double de fidélité et de trahison, Celan l'exprime avec une force remarquable dans ces traductions. Cela tient sans doute au rapport interne de Celan à sa propre langue - langue qui précisément depuis la Shoah ne peut plus lui être que souffrante désappropriation -, et qui ne peut en rien se mesurer à la relation que Cayrol entretient à la sienne. Entre la langue du traducteur et celle de l'écrivain, une irréductible dissymétrie éloigne et sépare, et la traduction s'affranchit de sa supposée secondarité, elle devient plus originaire que le texte dit original : la traduction bouleverse l'ordre du texte, le cours * normal » des choses, elle dira plus et mieux dans l'après coup - même en se taisant et en s'effaçant - que le poème de Cayrol. On comprend alors que cette traduction offre au lecteur le lieu aussi rare que raréfié de l'élaboration psychique de la perte, à jamais sans nom. Ainsi, les traductions-poèmes de Celan, dunt Nouss commente plusieurs décisions (qui tombent presque comme des verdicts) en assumant à son tour le transfert de la traduction, réintègrent $\alpha$ ce qui avait été lajssé tomber dans le texte de Cayrol : la traduction non seulement laisse le refoulé revenir dans le dire - mais aussi dans l'impossible à dire, ce qui ne cesse de s'inscrire/s'effacer par atténuation, retrait, matité, disparition.

A déchiffrer ces traductions de Celan avec la patience et l'attention douloureuse qu'elles commandent, on se rend vite compte que, si elles dérogent aux règles convenues (et ne viendrait même pas l'idée de parler ici de lapsus ou d'erreurs), c'est qu'elles cherchent ainsi à demeurer fidèles à une autre Loi, plus élevée et plus exigeante. Devant une telle expérience, il est également évident que certaines frontières, trop vite

${ }^{38}$ Jacques Derrida, « Fidélité à plus d'un ", Cahiers Intersignes, ${ }^{\circ} 13$, automne 1998, p. 224. 
érigées par certains théoriciens, entre la traduction et le commentaire sont en réalité impraticables. De fait, ces traductions-poèmes relèvent de manière si claire d'une lecture-écriture qu'elles démentent tout cloisonnement, toute séparation hiérarchisante entre commentaire et traduction. A la position tranchée et dogmatique de Laplanche par exemple qui pose que * l'un et l'autre ne devraient jamais être confondus ${ }^{39}$ "et réserve à chacun un temps distinct, on préférera, et de loin, celle de Jacques Derrida, qui souligne au contraire leur proximité et leur enchevêtrement :

[...] a a mes yeux les traducteurs sont les meilleurs lecteurs. Et méme les seuls lecteurs si on tient, comme je suis tenté de le faire, que le lecteur vigilant est attentif, toujours, à ce qui, dans un texte, travaille au corps idiomatique de la langue, s'y expose et dissimule en ces lieux où la traduction parait à la fois refusée et par là même appelée. Quiconque lit un texte, le lit bien, en prêtant toute l'attention requise a l'idiome, au travail d'écriture, à la singularité de la composition, etc. est en position de traducteur, déjà en train d'éprouver, pour s'y mettre à l'épreuve, la résistance d'une écriture pensante, poétique et idiomatique. [...] Dans le corps-à-corps avec le texte, ils identifient aussi les faiblesses, les contingences, les autres possibilités laissées en sommeil par une interruption ${ }^{40}$.

Cette image du traducteur comme veilleur-éveilleur de langues convient admirablement à l'expérience singulière de Celan, mais elle pourrait également éclairer ce qui se passe dans toute traduction " réussie ", c'est-à-dire porteuse d'intuitions poétiques ou d'Einfallen analytiques (c'est d'ailleurs, très rigoureusement, du même ordre), qui tente $d$ 'inventer une autre pensée de la traduction, une traduction pour laquelle la plus grande infidélité ne logerait pas dans le risque de la " dérive " interprétative ou dans la tentation du commentaire inavouée, mais dans la neutralisation de la charge poétique de l'écriture et qui

${ }^{39}$ Jean Laplanche, op. cit, p. 253. * Commenter, traduire, interpréter, faire travailler, sont des temps distincts, à respecter dans leur indépendance $[\ldots]$ ".

${ }^{40}$ Jacques Derrida, « Fidélité à plus d'un ”, Cahiers Intersignes, $n^{\circ} 13$, automne 1998, p. 251. 
oublierait que tout, toujours, " se joue au plus serré qui soit, à la nuance et dans l'absolument infinitésimal ${ }^{41}$.

Il y aurait beaucoup à dire sur cette relation du traducteur à sa langue, relation qui surdéterminera sans doute des transferts traductifs très différents selon qu'on définisse la langue en termes d'appropriation ou dans sa divisibilité inteme à elle-même. Lorsque, par exemple, Pontalis déclare qu' * Un auteur pense dans sa langue, il y a tension (* ce n'est pas ça que je veux dire "), il n'y a pas de hiatus. Le traducteur, lui, réside dans ce hiatus ${ }^{42}$ ", une telle distinction suppose non seulement une séparation entre l'auteur et le traducteur, renvoyés dos à dos chacun de leur côté de la frontière, mais aussi que le traducteur, « une fois déporté dans la langue étrangère " (et le mot porte déjà avec lui de toutes autres connotations que,

${ }^{41}$ Kadhim Jihad, a Pour Derrida (en le traduisant) „, Cahiers Intersignes, $\mathrm{n}^{\circ} 13$, automne 1998, p. 213. L'auteur témoigne de manière très intéressante de son expérience de traducteur du texte derridien en arabe et donne l'exemple, qui s'est avéré partículièrement difficile, du mot * différance ", concept qui configure, comme on le sait, dans sa graphie même la pensée de la trace. Jihad a opté pour le mot $\alpha$ Ikh(ti)lâf $*:$ Lu sans les parenthèses, le mot signifie différence. Lu en prenant en compte les parenthèses qui neutralisent ou ajournent, different, ce qu'elles contiennent, le mot devient : Ikhtâf, ce qui signifie ajournement, retardement, report, ne pas être au rendez-vous, voire être en désaccord, en discorde, en discordance, en différend. * Trouvaille pour les uns, agression pour les autres, la solution introduisait un corps étranger dans le système de la langue arabe puisque, souligne Jihad, a c'est la première fois que quelqu'un impose des parenthèses à double effet ou pour double lecture à l'intérieur d'un mot arabe, les parenthèses étant utilisées uniquement pour les phrases ou parties de phrases. L'unité du mot étant encore tenue pour intouchable, c'est au corps de la langue, à sa chair, qu'on aura ici touché. Dites-moi ce que vous avez fait de vos fétichismes, je vous dirai qui vous êtes " (ibid., p. 216). Bel exemple de ce que l'interdit et la transgression ne passent pas par les mêmes points d'une langue à l'autre. D'ailleurs, les traducteurs de Lecture de Derrida de Sarah Kofman déplaceront les parenthèses, écrivant $l k h t i l(a) f$, pensant sans doute rendre mieux service au $a$ derridien, mais ne se rendant pas compte qu'ils rendaient ainsi le mot arabe complètement inoperrant. Comme quoi une traduction peut toujouss en refouler une autre...

${ }^{42}$ J.-B. Pontalis, op. cit., p. 199. 
disons, " transporté " ou " déplacé "), entretient un rapport nécessairement abaissé à sa langue : l'autre langue lui apparaît toujours a plus "riche", plus "nuancée", plus "imagée", plus "musicale" "que la sienne, sans chair, squelettique, exsangue ${ }^{43} n$. Dévalorisation, voire haine de sa langue maternelle, survalorisation de la langue étrangère : le traducteur oscille selon Pontalis de manière ambivalente entre ces deux extrêmes, écartelé entre les deux langues, mais peut-être ne voit-il pas suffisamment à quel point la langue dite d'origine est elle-même écart par rapport à elle-même (contrairement à ce qu'il dit, tout écrivain se fait très littéralement le traducteur de ce hiatus interne). On entrevoit également qu'une telle façon d'envisager la traduction comme coupure désormais infranchissable entre un avant absolu et un après où, tout au mieux, le traducteur n'a d'autre choix que d'errer de * compromis en compromis, d'à-peu-près en à-peuprès $^{44}$ n, ne puisse engendrer que nostalgie, mélancolie, endeuillement irressolu et insurmontable - ce qui n'est pas incompatible avec une certaine érotisation/sublimation de la perte. A entendre les accents plaintifs qui caractérisent la position de Pontalis sur la traduction, on peut en effet se demander si cette " mélancolie du langage " ne serait pas une manière d'entretenir encore, contre l'expérience analytique, une illusion quant à la langue originale/originelle tenue pour une et intacte, non divisée, non entamée par l'Autre.

Or il ne fait pas de doute que le transfert traductif s'oriente dans une tout autre voie si cette " origine " est elle-même tenue pour une "prothèse ", toujours déjà hôte de l'Autre et en écart par rapport à ellemême. On reconnaîtra ici la position de Jacques Derrida lorsqu'il évoque la situation du monolingue - autre nom possible du traducteur - qui

${ }^{43} \mathrm{Ibid} ., \mathrm{p}, 200$. On peut se demander si ce rejet de la langue maternelle chez Pontalis ne nourrit pas le « Moi idéal n du traducteur, sévèrement stigmatisé s'il laisse a transparaître le moindre indice de la langue source "(Julia Kristeva, * L'Autre langue, ou traduire le sensible ", l'Infini, n 57, printemps 1997, p. 16). On pourrait le croire à entendre le dégoût qui s'exprime dans ces propos : « Pour moi, "faux sens" et même "contresens" décelés dans une traduction sont péchés véniels. Mais pouvoir dire : ̧a sent la traduction, cela, c'est rédhibitoire. [...] Les odeurs de traduction, c'est comme les odeurs de cuisine : ça coupe l'appétit et ôte au mets sa saveur * (op. cit., p. 202).

${ }^{44}$ Ibid., p. 198. 
entretient de naissance un rapport complexe à plus d'une langue (ce qui distinguerait déjà sa situation de celle de Pontalis, encore assuré de pesséder sa langue maternelle : le monolingue derridien n'est pas dans le manque de la langue d'origine, sa restauration ou sa réparation, son devoir de mémoire est tout autre) :

Le monolingue dont je parle, il parle une langue dont il est privé. Ce n'est pas la sienne, le français. Parce qu'il est donc privé de toute langue, et qu'il n'a plus d'autre recours - ni l'arabe, ni le berbère, ni l'hébreu, ni aucune des langues qu'auraient parlées des ancêtres -, parce que ce monolingue est en quelque sorte aphasique (peut-être écrit-il parce qu'il est aphasique), il est jeté dans la traduction absolue, une traduction sans pôle de référence, sans langue originaire, sans langue de départ. Il n'y a pour lui que des langues d'arrivée, si tu veux, mais des langues qui, singulière aventure, n'arrivent pas à s'arriver, dès lors qu'elles ne savent plus d'où elles partent, à partir de quoi elles parlent, et quel est le sens de leur trajet. Des langues sans itinéraire. ${ }^{45}$

Cette situation du traducteur monolingue, jeté cette fois dans la " traduction absolue " parce que la première langue est non pas seulement " perdue " (au sens du manque et du désir nostalgique évoqués par Pontalis), mais défout originaire, appelle une conception différante de la traduction ${ }^{46}$ qui insistera sur la " non-identité à soi de toute langue ${ }^{47}$ ". Pour Derrida, "Une langue n'existe pas. [...] Ni l'idiome ni le dialecte ": le monolingue traducteur " ne fait jamais un avec lui-même $e^{48}$, il doit " fidélité à plus d'un ". Qu'est-ce à dire ? Tout simplement (mais prendre en considération ce fait bouleverse complètement les certitudes de la linguistique classique ou de la traduction * appliquée "), que s'engage, avec cette fantasmatique du traducteur aphasique, une autre pensée de la traduction, " sans langue originaire, sans langue de départ ". "Traduction

${ }^{45}$ Jacques Derrida, Le monolinguisme de l'autre ou La prothèse d'origine, Paris, Galilée, « Incises ", 1996, p. 117. C'est l'auteur qui souligne.

${ }^{46}$ Voir sur ce sujet ie collectif Tradução. A prática da diferença, sous la direction de Paulo Ottoni, Campinas (Brésil), Editora da Unicamp, FAPESP 1998, 159 p.

${ }^{47}$ Jacques Derrida, op. cit., p. 123.

11 Ibid., p. 123. 
d'une langue qui n'existe pas encore, et qui n'aura jamais existé, dans une langue à l'arrivée donnée $e^{49}$, ce qui implique une traduction interne se produisant à l'intérieur même de la langue, dans le rapport à soi de la langue. Comme le rappelait encore récemment Derrida, l'idiome dans cette perspective « n'est jamais le propre ou l'identité à soi du propre, il est déjà différent de lui-même, il n'est qu'en différence. Il inscrit un écart en lui-même. Cet écart conditionne son économie irréductible dans une langue en même temps qu'il annonce, sans attendre, à cause de l'écart, le travail de traductibilité. Déjà au-dedans d'une seule langue ${ }^{50}$ ». La déconstruction - dont Derrida n'a jamais voulu donner une définition, à laquelle il a sans cesse donné * plus d'un * nom, et de plus en plus souvent ces dernières années, sans le théoriser " comme tel ", celui de traduction - apparaît à ce titre comme un indispensable chaînon manquant (l'anglais dit mieux * missing link *) pour repenser cette relation du traducteur comme « être avec " la langue, la sienne (quoi que puisse vouloir encore approprier ce possessif) et celle de l'autre (dont il est l'hôte et l'otage, être avec soi sans jamais être-chez-soi).

Qu'une traduction puisse inférer des effets poétiques, comme c'est manifestement le cas dans le travail de Celan, qu'elle puisse même être reçue comme la forme la plus vigilante d'écoute du texte et formuler à travers ses signifiants en souffrance une véritable interprétation analytique, cette possibilité découle donc d'un certain rapport du traducteur à sa propre langue, de sa capacité à y entendre l'inquiétante étrangeté, l'étrange/familier qui y loge déjà. À défaut de pouvoir explorer de manière approfondie dans le cadre du pressent texte les retombées d'une telle philosophie de la traduction - qu'on pourrait également qualifier, sans la dénaturer je crois, de poétique - qui ouvre à coup sûr une nouvelle voie, traduction de l'écriture qui appelle en retour à inventer une autre écriture de la traduction, je souhaitais tout au moins marquer l'importance de cette question de la traduction interne et souligner comment elle éclairait à la fois la traduction poétique et la lecture psychanalytique.

49 Jbid., p. 123.

${ }^{36}$ Jacques Derrida, "Fidélité à plus d'un *, Cahiers Intersignes, $n^{\circ} 13$, automne 1998, p. 224. 
On le voit : le traducteur en psychanalyse fait immanquablement l'épreuve de la perte, du manque certes, mais tout aussi bien, et peut-être plus significativement encore, celle du passage même. Et ce traducteur en psychanalyse, on peut le saisir à des paliers très différents de son parcours : en traducteur professionnel qui se penche sur un texte psychanalytique, en traducteur freudologue qui sera peut-être (ou non) passé par l'expérience de la cure, en traducteur-lecteur qui sera capable d'analyser son contre-transfert au texte ou, plus radicalement encore, en écrivain, cet étranger-traducteur qui s'attache à * traduire le sensible ", comme le définit Julia Kristeva :

Cette langue sensible n'est pas une langue de signes : elle est une " langue " entre guillemets, un chaos et un ordre de battements, d'impressions, de douleurs et d'extases aux frontières de l'informulable biologie. Cette " langue n-là est la véritable * étrangetén - plus étrangère que tout idiome déjà constitué - que l'écrivain espère formules!'.

Les voies de traverse entre psychanalyse et traduction sont - cette livraison l'illustre clairement - fort diverses, les unes balisées, les autres beaucoup plus imprévisibles. Plusieurs textes de ce numéro - ceux de Christie McDonald ${ }^{52}$, de Simon Harel et de René Major me viennent tout particulièrement à l'esprit ici - ont poursuivi l'exploration du thème en lui donnant son extension, son pouvoir d'effraction les plus grands, les plus étonnants. Dans chacune des scènes d'écriture qui les ont retenus, la psychanalyse et la traduction se sont rencontrées en une * fiction-vérité *, selon le mot de René Major, au plus près de la réalité singulière de

${ }^{s 1}$ Julia Kristeva, art. cité, p. 23.

${ }^{52}$ Sans qu'elle ait été concertée, une convergence émouvante se dessine entre ce texte portant sur Sarah Kofman et celui d'Alexis Nouss sur Celan, tous deux de manière indélébile marqués par l'événement de la Shoah (qui ne s'est pas passé, qui n'a cessé d'arriver pour eux jusqu'à la toute fin de leur ceuvre). Sarah Kofman écrivait au fronton de cette autobiographie qui devait être son legs final, son ultime signe d'écrivain : " Mes nombreux livres ont peut-être été des voies de traverse obligées pour parvenir à raconter "ça" „ (Rue Ordener, rue Labat, Paris, Galilée, 1994). 
l'expérience analytique. Ces rencontres se donnent d'ailleurs le plus souvent à lire sur le mode du silence et de l'évitement, comme ce sera le cas dans la relation transférentielle " mutuelle entre Beckett et Bion, traduisant l'un l'autre leur expérience de la cure dans une ouvre fictionnelle, figurant plus étrangement encore l'un pour l'autre une sorte de jumeau imaginaire; du retrait et de l'ambivalence, notamment dans l'écriture de soi qui fut pour Sarah Kofman une ultime épreuve de traduction $d$ " "elle-même ", elle qui avait placé la traduction au croisement de la philosophie, de la psychanalyse et de la littérature, la rendant présente à tous les carrefours de sa réflexion théorique ${ }^{53}$; ou encore du secret, à la fois voilé et gardé, échangé à travers des lapsus calami qui se donnent à lire - à entrelire, ou à entrevoir plutôt - dans deux textes récents d'Hélène Cixous et de Jacques Derrida réunis sous un titre commun, Voiles. C'est par cette énigmatique scène à trois (car le lecteur-traducteur-interprète est lui aussi inscrit dans la scène du déchiffrement) que se clôt cette livraison, et on ne saurait faire sentir avec une plus grande délicatesse que René Major ce qui fait rêver dans le passage entre les langues, entre les textes, entre les différences (toujours sexuelles aussi) au moment où la rencontre textuelle entre psychanalyse et traduction a lieu et prend corps. Pratique elle-même exemplaire de la détraduction/retraduction opérée par l'écoute analytique, la lecture de René Major travaille tout autant à dévoiler qu'à revoiler la proximité de ces deux textes dans leur étrangeté même : dans ces lapsus calami-ces " caractères cryptographiques que nous ne nous donnons pas la peine de déchiffrer ${ }^{54}$, déplorait Proust - un transfert traductif, un contact parlant a bien lieu, à l'insu et à l'invu des deux écrivains et de leur traducteur témoin. Et ce n'est certes pas un hasard si cette rencontre, scène de

${ }^{53}$ On mentionnera à ce titse la position de tiers traducteur qu'elle occupera si fréquemment entre Freud et Nietzsche, ses deux écrivains de prédilection, qu'elle ne cessera jamais de traduire dans leur proximité et leur différence l'un à l'autre.

${ }^{34}$ Proust invoque dans ce passage la * vérité qu'on n'aperçoit pas $*$, mais que * nous sentons à l'intérieur * quand les impressions * nous aiguillonnent de la pointe de vérité ; : « Et ceci sa réalité passe à notre seuil et nous laisse une note sur elle en caractères cryptographiques que nous ne nous donnons pas la peine de déchiffrer " (Matinée chez la Princesse de Guermantes, Henti Bonnet et Bernard Brun (éditeurs), Paris, Gallimard, p. 327, cité par Julia Kristeva, art. cité, p. 23). 
séduction ${ }^{55}$ qui a lieu sans avoir lieu, qui passe sous (ou par-dessus) les mots, brouille en retour les lignes de partage convenues entre vérité et fantasme, autobiographie, témoignage et fiction, pour atteindre à cette " fiction-vérité " plus réelle que celle de la réalité. Dans chacune de ces expériences d'écriture-lecture, la traduction et la psychanalyse - mais il s'agit là d'une tout autre relation encore : de la psychanalyse en traduction - sont bien en effet conjointes l'une à l'autre, traversées de l'autre quel que soit le sens de cette provenance ou de cette arrivée."

\section{Université de Montréal}

\section{Références}

BENJAMIN, Walter (1991)." La tâche du traducteur ». Traduction par Martine Broda. Poésie, $n^{\circ} 5$.

BENSLAMA, Fethi (1998). « La répudiation originaire ^. Cahiers Intersignes, * Idiomes, nationalités, déconstructions. Rencontre de Rabat avec Jacques Derrida $n, n^{\circ} 13$.

BERMAN, Antoine (1984). L'Épreuve de l'étranger. Culture et traduction dans l'Allemagne romantigue. Paris, Gallimard, * Les Essais *.

DERRIDA. Jacques (1998). * Fidélité à plus d'un ». Cahiers Intersignes, $n^{\circ} 13$.

- (1996). Le Monolinguisme de l'autre ou La prothèse d'origine. Paris, Galilée, * Incises *.

53 Cette scène de séduction fait écho à d'autres expériences de séduction freudiennes également évoquées dans ce numéro par Patrick J. Mahony et Monique Schneider, qui remarquent tous deux à quel point tout ce qui touche l'Urheberin (l'initiatrice en matière sexuelle) et l'Urherber (le générateur) - autrement dit, le passage de la différence sexuelle - pose problème à la traduction.

- Je tiens à remercier vivernent Patrick Poirier de l'aide inestimable qu'il m'a donnée dans la préparation matérielle de ce numéro. Je le remercie également d'avoir bien voulu assurer la traduction du texte de Patrick J. Mahony. 
- (1976). « Fors ^. Dans Nicolas Abraham et Maria Torok, Cryptonymie. Le Verbier de l'Homme aux Loups. Paris, Flammarion, "Champs ".

GUBRICH-SIMITIS, Ilse (1991). * Histoire de l'édition des œuvres de Freud en langue allemande $»$. Revue internationale d'histoire de la psychanalyse, 4, p. 13-71.

JIHAD, Kadhim (1998). " Pour Derrida (en le traduisant) ". Cahiers Intersignes, $\mathrm{n}^{\circ} 13$.

KOFMAN, Sarah (1994). Rue Ordener, rue Labat. Paris, Galilée.

KRISTEVA, Julia (1997), " L'autre langue, ou traduire le sensible ". L'Infini, $\mathrm{n}^{\circ} 57$.

LAPLANCHE, Jean (1997). Le primat de l'autre en psychanalyse. Travaux 1967-1992. Paris, Flammarion, “ Champs *.

MICHAUD, Ginette (1988), * Freud : N.D.T. n. Etudes françaises, 24 :3.

MICHAUD, Ginette (1989). * Freud : N.D.T. ou Affects et fantasmes chez les traducteurs de Freud ", TTR, II : 2.

PERALDI, François (1982). * Psychanalyse et traduction *. Meta, $27: 1$.

PONTALIS, J.-B. et Jean LAPLANCHE (1967). * Connaître Freud avant de le traduire n. Le Monde, $\mathrm{n}^{\circ} 6884$.

- (1988). Perdre de vue. Paris, Gallimard, * Connaissance de l'Inconscient $n$.

ROUDINESCO, Elisabeth et Michel PLON (1997). Dictionnaire de la psychanalyse. Paris, Fayard.

STEINER, Riccardo (1991). "Une marque internationale universelle d'authenticité. Quelques observations sur l'histoire de la traduction anglaise de l'œuvre de Sigmund Freud, en particulier sur les termes techniques ". Revue internationale d'histoire de la psychanalyse, 4, p. 71188. 
Tradução. A prática da diferença (1998). Sous la direction de Paulo Ottoni, Campinas (Brésil), Editora da Unicamp, FAPESP.

RÉSUME : Psychanalyse et traduction : voies de traverse L'auteure présente les diverses voies d'approche abordées dans cette livraison, de la traduction $d u$ texte psychanalytique, aux transferts du traducteur freudien, en passant par la psychanalyse en traduction (lorsque la psychanalyse se fait elle-même opération traduisante entre les discours). A partir de réflexions en forme d'associations libres, elle interroge également certaines positions de Jean Laplanche, J.-B. Pontalis et Jacques Derrida qui éclairent les échanges se produisant entre psychanalyse et traduction.

ABSTRACT : Psychoanglysis and Translation : Passages Between and Beyond - The author presents the different approaches dealt with in this issue : first, the translation of psychoanalytic texts; then, the transfers of Freud's translators; and, finally, psychoanalysis in translation (when psychoanalysis is, itself, an act of translation between disocurses). Through reflections in the form of free associations, the author goes on to question the positions taken by Jean Laplanche, J.-B. Pontalis, and Jacques Derrida, thereby clarifying the reciprocal exchanges between psychoanalysis and translation.

Ginette Michaud : Département d'études françaises, Université de Montréal, C.P. 6128, Succursale Centre-ville, Montréal (Québec) H3C 3J7. 